\title{
Medición volumétrica de la cavidad acetabular en pacientes con displasia del desarrollo de la cadera luxada inveterada unilateral operados en un solo tiempo
}

\author{
Volumetric measurement of the acetabular cavity in patients with unilateral neglected \\ developmental dysplasia of the dislocated hip operated in a single time
}

Benjamín Araujo-Monsalvo', Alejandro Trujillo-Satow², Víctor M. Araujo-Monsalvo³, Ramiro Cuevas-Olivo², Luis M. Hernández-Simón y y Víctor M. Domínguez-Hernández 3 ,4*

${ }^{1}$ Departamento de Ingeniería de Sistemas, Sección de Estudios de Posgrado e Investigación, Escuela Superior de Ingeniería Mecánica y Eléctrica, Instituto Politécnico Nacional; ${ }^{2}$ Servicio de Ortopedia Pediátrica, Instituto Nacional de Rehabilitación "Luis Guillermo Ibarra Ibarra"; ${ }^{3}$ Laboratorio de Biomecánica, Instituto Nacional de Rehabilitación "Luis Guillermo Ibarra Ibarra"; ${ }^{4}$ Tecnologico de Monterrey, Campus Ciudad de México. Ciudad de México, México

\begin{abstract}
Resumen
Antecedentes: El método más utilizado para la evaluación posquirúrgica de la displasia del desarrollo de la cadera inveterada es la radiografía convencional, lo que impide medir con precisión cambios intraarticulares. Objetivo: Analizar los cambios morfológicos del volumen acetabular mediante tomografía computarizada en pacientes operados con miotomía de aductor mediano, tenotomía de psoas, reducción abierta de cadera, diafisectomía, osteotomía varizante y desrotadora, y acetabuloplastia tipo Dega. Método: Se realizó un estudio piloto en el que se analizaron tomografías de seis pacientes del Instituto Nacional de Rehabilitación con displasia inveterada unilateral, por medio de software de diseño para determinar el volumen acetabular. Resultados: Se encontró que el volumen acetabular operado fue mayor que el contralateral (8 vs. 48\%), pero no se halló diferencia significativa entre los volúmenes $(p=2.46)$. Se encontraron diferencias significativas entre los volúmenes y la edad de los pacientes, evaluadas mediante ANOVA multifactorial $(p=0.006)$, Cochran $\left(p=2.925 \times 10^{-8}\right)$ y Kruskal-Wallis $(p=0.00019)$. Conclusión: Es claro que la técnica de medición propuesta permite identificar diferencias entre volúmenes (operado y contralateral). El seguimiento clínico de estos pacientes podrá validar los resultados obtenidos por este método y servirá para poder estimar la eficacia de la técnica quirúrgica aplicada en los pacientes.
\end{abstract}

PALABRAS CLAVE: Cavidad acetabular. Displasia del desarrollo de la cadera inveterada. Estudio tomográfico. Medición volumétrica. Técnica Dega.

\section{Summary}

Background: Plain radiography is the most often imaging method used for postoperative assessment of inveterate developmental dysplasia of the hip. This technique does not permit to achieve an accurate diagnose of intra-articular changes, specifically articular congruity. Objective: analyze the morphological changes of the acetabular volume by computed tomography, in patients operated by medium adductor myotomy, psoas tenotomy, open hip reduction, diaphysectomy, varus osteotomy and derotating and Dega-type acetabuloplasty. Method: A pilot clinical trial was conducted including six patients with unilateral inveterate dysplasia admitted to the Rehabilitation National Institute. Computed tomography were analyzed using an engineering design software. Results: It was found that in all cases analyzed, operated acetabular volume was greater than the

\footnotetext{
Correspondencia:

*Víctor M. Domínguez-Hernández

Calz. México-Xochimilco, 289

Col. Arenal de Guadalupe, Del. Tlalpan

C.P. 14389, Ciudad de México, México

E-mail: vm_dominguez@yahoo.com.mx
}

Fecha de recepción: 05-05-2018

Fecha de aceptación: 23-04-2019

DOI:10.24875/CIRU.19000436
Cir Cir. 2019;87:490-495

Contents available at PubMed www.cirugiaycirujanos.com 
contralateral (8 to 48\%), however, no significant difference between the volumes was found ( $p=2.46)$. Significant difference between the volume and the age of patients, in multifactorial ANOVA tests $(p=0.006)$, Cochran's $(p=0.00019)$ and Kruskal-Wallis $\left(p=2.925 \times 10^{-8}\right)$ was found. Conclusion: It is clear that the proposed measurement technique is able to identify differences between volumes (operated and contralateral). The clinical monitoring of these patients can validate the results obtained by this measurement technique, and it will serve to estimate the effectiveness of the surgical technique applied in patients.

KEY WORDS: Acetabulum. Inveterate developmental dysplasia of the hip. Tomography study. Volumetric measurement. Dega technique.

\section{Introducción}

La displasia del desarrollo de la cadera es una patología de etiología multifactorial, tanto por predisposición genética como por factores mecánicos intrínsecos y extrínsecos ${ }^{1,2}$. Cuando esta patología no se diagnostica y trata tempranamente, puede generar cambios estructurales anatómicos en la cadera. En la población mayor de 3 años (luxación inveterada) los cambios se caracterizan por desplazamiento alto de la cabeza femoral, insuficiencia del acetábulo y aumento de la anteversión en el cuello femoral ${ }^{3}$.

El tratamiento de la displasia del desarrollo de la cadera luxada en los niños mayores de 3 años es complicado, ya que se requieren varios procedimientos para obtener una reducción concéntrica estable. Debido a las tres condiciones existentes en las caderas de estos pacientes (luxación alta, anteversión femoral aumentada y displasia acetabular), el tratamiento ideal recomendado es su corrección en un solo procedimiento. Es aceptado de manera universal que para las luxaciones altas lo recomendable es la realización de diafisectomía femoral con o sin varización o desrotación. Sin embargo, no existe un acuerdo respecto al tratamiento de las displasias acetabulares en este grupo de edad. Hay una amplia variedad de técnicas quirúrgicas descritas para tratar la displasia acetabular residual en la displasia del desarrollo de la cadera. Sin embargo, la técnica Dega es una de las osteotomías que permite no solo reorientar, sino también dar forma al acetábulo.

Se ha descrito que las acetabuloplastias periacetabulares, como la técnica Dega, reducen el tamaño del acetábulo, por lo que los cambios estructurales posquirúrgicos sobre el acetábulo pueden conducir a cambios degenerativos articulares (pérdida de la congruencia esférica) $)^{4-10}$.

La tomografía computarizada tridimensional (3D) proporciona una visualización completa de la cadera, con la cual es posible hacer una reconstrucción, y con ello valorar la anteversión femoroacetabular. Los modelos 3D permiten estimar la cobertura y medir la superficie y el volumen acetabular, con lo que se puede tener un diagnóstico más preciso, y de esta forma poder indicar un tratamiento adecuado para la displasia de cadera inveterada ${ }^{11-13}$.

Es cierto que ningún estudio cumple con la precisa y exacta posición del acetábulo al momento de la reconstrucción espacial de la pelvis. Las razones son la falta de estándares, planos de referencia estables de la pelvis y un método de medición independiente basado en la antropometría ${ }^{14-18}$, donde se evalué la maniobra empleada para el desplazamiento del fragmento (osteotomía), para evitar posiciones no deseadas del acetábulo, tales como una excesiva rotación externa, retroversión del acetábulo o una cobertura posterior insuficiente de la cadera ${ }^{19}$.

Por ello, el objetivo de este artículo es la presentación de un método para la medición del volumen acetabular de pacientes mayores de 3 años con displasia del desarrollo de la cadera luxada inveterada unilateral, por medio de tomografía computarizada 3D posoperatoria, así como la comparación del acetábulo operado contra el contralateral, con la finalidad de observar cambios morfológicos que permitan, dependiendo de la edad del paciente y del momento de realización del procedimiento quirúrgico, la posible normalización del acetábulo operado.

\section{Método}

Se realizó un estudio piloto basado en criterios internos del Instituto Nacional de Rehabilitación para la selección de los pacientes. Los criterios para la selección fueron los siguientes: pacientes de cualquier sexo, mayores de 3 años, con cadera luxada, no traumática, y displasia acetabular, sin tratamiento médico ni quirúrgico previo, e ingreso al protocolo de tratamiento con consentimiento informado firmado por sus tutores. 

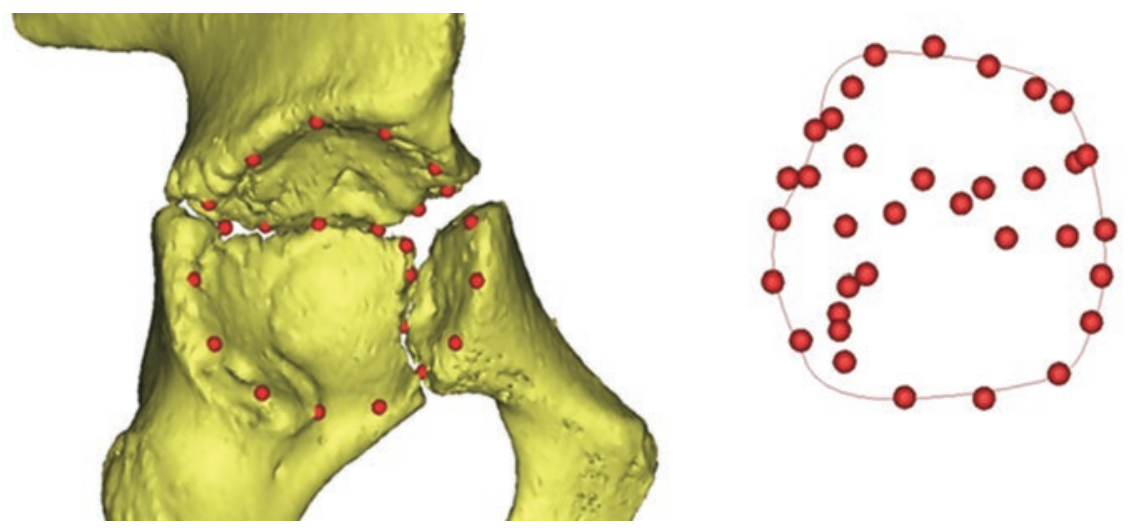

Figura 1. Creación de puntos en el contorno y en el interior del acetábulo como referencia para el análisis y la medición del volumen.

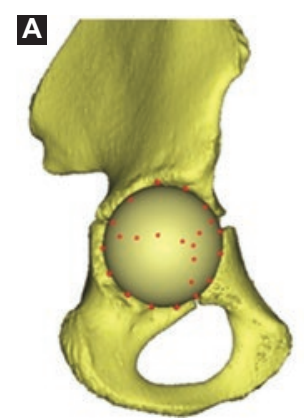

B

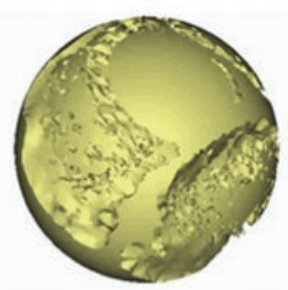

C

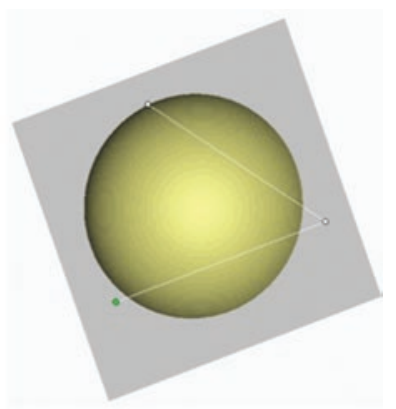

Figura 2. A: Creación de una esfera a partir de cuatro puntos seleccionados de la circunferencia del acetábulo. Cobertura total del acetábulo. B: Operación booleana: resta de la imagen y de la pelvis; generación de objeto con interior del acetábulo. C: Creación de un plano cubriendo las crestas del acetábulo trirradiado.

Se seleccionaron seis pacientes, en el periodo 2003-2006, en el servicio de ortopedia pediátrica del Instituto Nacional de Rehabilitación. Se revisaron las historias clínicas y los estudios tomográficos 3D de los seis pacientes. Este estudio se realizó utilizando imágenes obtenidas del sistema de información médica y telemedicina PACS-INR (Picture and Communication System del Instituto Nacional de Rehabilitación Luis Guillermo Ibarra Ibarra).

El procedimiento quirúrgico consistió en miotomía del primer aductor, tenotomía de psoas por abordaje inguinal tipo Ludloff, diafisectomía varizante y desrotadora, reducción abierta por abordaje anterior de Smith-Petersen y acetabuloplastía tipo Dega, con seguimiento posoperatorio mínimo de 2 años.

Las tomografías computarizadas se realizaron con un equipo multicorte GE Light Speed de 64 cortes (GE Light Speed, General Electric Company, Fairfield, CT, EE.UU.), área azul con estación de trabajo y visualizadores de imagen. Los criterios del estudio tomográfico consistieron en un estudio transversal, de estratificación interna (operado vs. contralateral), descriptivo y de simulación diacrónica, de pacientes con cadera luxada no traumática y displasia acetabular unilateral operados mediante la técnica descrita para la luxación, y displasia femoral, con acetabuloplastia de Dega modificada, edad mayor de 3 años y seguimiento posquirúrgico mínimo de 2 años.

Se obtuvieron imágenes en formato DICOM (Digital Imaging and Communication in Medicine) a partir de un estudio tomográfico y se procesaron en el software Mimic's Innovation Suite (Materialise Inc., Leuven, Bélgica), versión 17. Se realizó una reconstrucción 3D de la pelvis para seleccionar la zona de trabajo. Por medio del software se crearon puntos en el contorno y el interior del acetábulo como referencia, unidos a través de una línea (Fig. 1), además de una esfera a partir de cuatro puntos seleccionados (Fig. 2 A). Posteriormente se realizó una operación booleana, con lo que se generó una esfera (objeto) con el interior del acetábulo (ilion, isquion y pubis) de manera detallada, y se creó un plano, colocándolo por arriba de las tres crestas de la pelvis (Fig. 2 B y C). Se realizó un corte ortogonal a la esfera, manteniendo la parte correspondiente al interior del acetábulo (Fig. 3). Se hizo una máscara de esta para seleccionar solo la zona de interés (limpieza). En la figura 4 se muestra la limpieza del interior del objeto, la cual se realizó con la presencia del cirujano, para poder delimitar la zona que se debe limpiar, y con la creación de polilíneas, para evitar la pérdida 

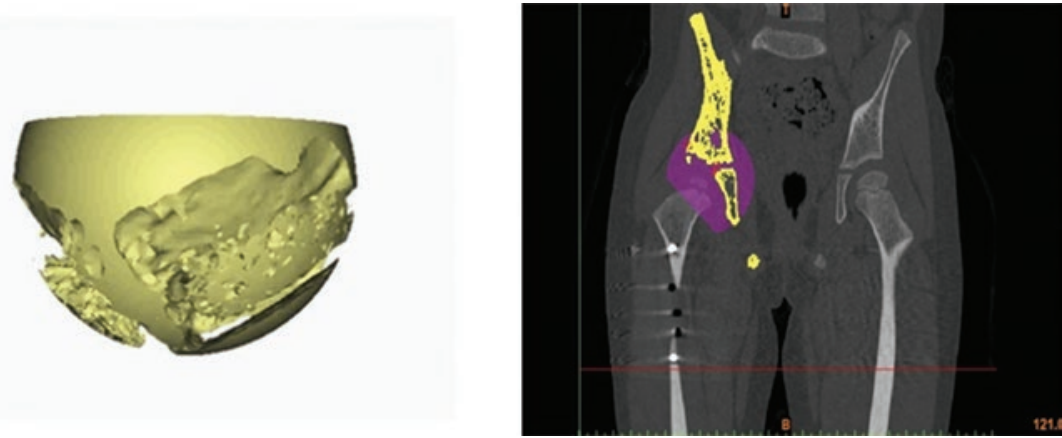

Figura 3. Corte de la esfera por medio del software y creación de la máscara de la zona de limpieza.
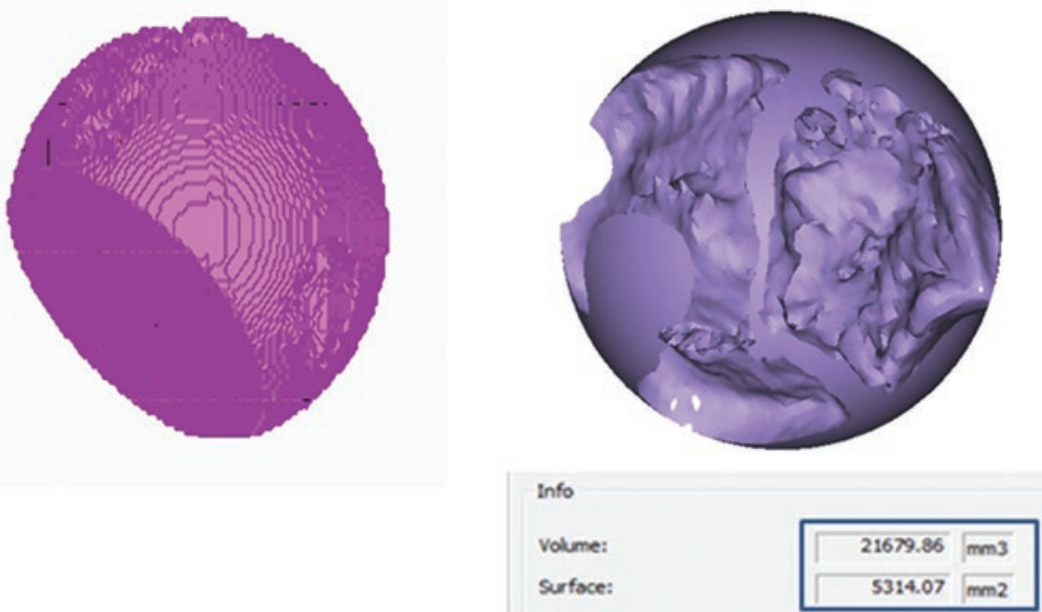

Figura 4. Limpieza del objeto resultante del corte ortogonal y medición del volumen por medio del software Mimics.

de información que afectara los resultados de la investigación. Por último, se midió el volumen del acetábulo. Las mediciones realizadas al acetábulo se recopilaron y se desarrollaron pruebas estadísticas con el software Statgraphics Centurion (Statpoint Technologies, Inc., Warrenton, Virginia, EE.UU.) versión 16.1.11; se aplicó la prueba de Kolmogorov-Smirnov para determinar la distribución de los datos.

Las diferencias entre los grupos para cada uno de los acetábulos (operado vs. contralateral) y la edad de los pacientes al momento de la cirugía (de 3 a 6 años) se evaluaron como muestras independientes utilizando la prueba ANOVA multifactorial, la comprobación de la varianza (Cochran) y la prueba de Kruskal-Wallis para la comparación de los grupos, con un nivel de confianza del $95 \%$ y un error del $5 \%$, además de un nivel de significancia de $p<0.05$.

\section{Resultados}

Se observó que en todos los casos el volumen del acetábulo operado fue mayor que el del acetábulo
Tabla 1. Resumen de medidas realizadas al acetábulo operado vs. el contralateral

\begin{tabular}{llcccc}
\hline Cirugía & \multicolumn{2}{c}{ Volumen $\left(\mathbf{c m}^{3}\right)$} & \multicolumn{2}{c}{ Diferencia } \\
\hline $\begin{array}{l}\text { Edad del } \\
\text { paciente (años) }\end{array}$ & Acetábulo Contralateral & Operado & $\mathbf{c m}^{3}$ & $\%$ \\
\hline 3 & Izquierdo & 12.82 & 13.95 & 1.12 & 8.81 \\
3 & Izquierdo & 11.35 & 13.85 & 2.50 & 21.96 \\
4 & Izquierdo & 14.75 & 16.24 & 1.48 & 10.04 \\
4 & Izquierdo & 19.55 & 21.68 & 2.12 & 10.89 \\
5 & Derecho & 17.05 & 19.75 & 2.70 & 15.79 \\
6 & Derecho & 10.06 & 14.83 & 4.78 & 47.53 \\
\hline
\end{tabular}

contralateral; la diferencia porcentual cambió dependiendo de la edad del paciente al momento de la cirugía, siendo más alta para el paciente de 6 años, seguida de un paciente de 3 años y el paciente de 5 años (Tabla 1).

Los datos muestran una distribución normal. Los valores de $p$ para cada elemento fueron $>0.05$. Se encontraron diferencias significativas en la prueba 


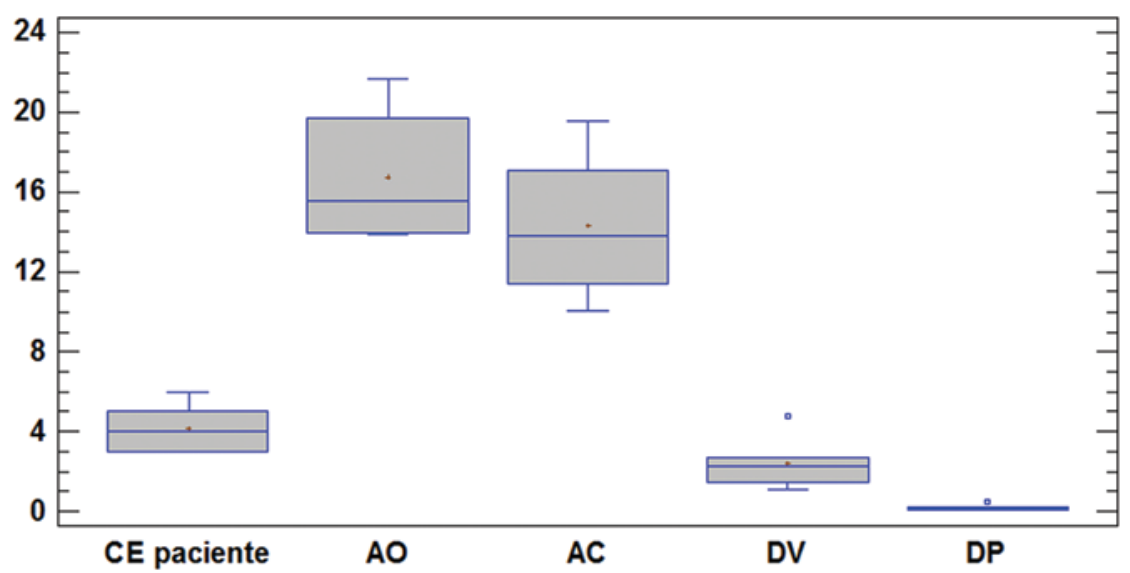

Figura 5. Comparación de las muestras por medio del software. AC: acetábulo contralateral; $A O$ : acetábulo operado; CE paciente: cirugía-edad del paciente; DP: diferencia porcentual; DV: diferencia en volumen.

ANOVA multifactorial con un valor de $p=0.006$. Las pruebas de comprobación de varianza (Cochran) y Kruskal-Wallis fueron significativas con $p=2.925 \times$ $10^{-8}$ y $p=0.00019$, respectivamente.

En cuanto a la prueba múltiple de rangos (LSD $95 \%$ ), no se encontró diferencia significativa entre el volumen acetabular operado y el contralateral, con $p=2.46$; entre volumen operado y la diferencia porcentual $(p=2.45)$; y entre volumen contralateral y diferencia porcentual $(p=4.91)$ (Fig. 5).

\section{Discusión}

Los procedimientos radiológicos se han utilizado para evaluar la orientación del acetábulo y son considerados como el estándar en imagen de la cadera, aunque no proporcionan información suficiente en los planos sagital y transversal ${ }^{20}$. Debido a lo anterior, los modelos tomográficos computarizados $3 \mathrm{D}$ surgen como opción de diagnóstico para evaluar las partes óseas de la pelvis, incluyendo los ángulos de anteversión del acetábulo, así como la evaluación de la geometría acetabular y el análisis biomecánico ${ }^{21,22}$.

La capacidad de reconstrucción por medio de tomografía computarizada 3D ha permitido la evaluación precisa de la gravedad de la displasia acetabular. Esta técnica de imagen puede contribuir a la evaluación radiográfica preoperatoria, mediante la cual es posible diagnosticar el tipo y el grado de deficiencia acetabular. En los pacientes con displasia del desarrollo de la cadera es necesaria la cirugía para lograr una congruencia satisfactoria entre la cabeza femoral y el acetábulo, para lo que se requiere un total conocimiento de la patología ósea de la displasia del desarrollo de la cadera, y de su estructura 3D, para planificar y seleccionar los procedimientos quirúrgicos adecuados.

La definición de volumen acetabular es importante. Si se define como el volumen de una esfera que hace el arco del techo acetabular de la circunferencia, el volumen acetabular disminuiría después de la osteotomía Dega, y la mayoría de los textos especializados parecen abogar por esta definición. Por otro lado, si la definición de volumen acetabular es la profundidad del acetábulo que contiene la cabeza femoral, entonces el volumen aumenta después de la osteotomía Dega. Creemos que esta definición es preferible, cuando se evalúa la cirugía pélvica, para la deficiencia acetabular del volumen acetabular ${ }^{23}$.

Los resultados con el método descrito en este artículo corroboran lo descrito por Chung, et al. ${ }^{24}$ y Ozgur, et al. ${ }^{7}$, quienes encontraron que, en los pacientes con displasia del desarrollo de la cadera, la osteotomía tipo Dega incrementa el volumen acetabular aproximadamente en un $50 \%$, reduciendo la presión intraarticular de la cabeza femoral.

La metodología presentada en este artículo simplifica la técnica de medición. Se basa únicamente en la evaluación de la geometría de las estructuras acetabulares, sin elementos como la cabeza y el cuello femoral, o la ubicación del cartílago trirradiado, y proporciona elementos suficientes para el análisis morfológico posoperatorio de los pacientes ${ }^{24}$.

En efecto, es necesario contar con estudios tomográficos prequirúrgicos para la realización de la técnica descrita, para poder comparar los acetábulos y los cambios morfológicos en el posoperatorio?.

Una de las principales limitaciones de este estudio es el reducido número de pacientes. Sería conveniente contar con una muestra mayor para tener más 
sensibilidad y obtener resultados que sean estadísticamente significativos, así como poder identificar los factores determinantes para un mejor diagnóstico y un tratamiento más eficaz.

\section{Agradecimientos}

Los autores agradecen el trabajo realizado por el técnico Martín Luna Méndez, del Servicio de Tomografía Computada del Instituto Nacional de Rehabilitación Luis Guillermo Ibarra Ibarra.

\section{Financiamiento}

Los autores declaran que no hubo fuentes de financiamiento.

\section{Conflicto de intereses}

Los autores declaran no tener conflicto de intereses relacionado con el presente manuscrito.

\section{Responsabilidades éticas}

Protección de personas y animales. Los autores declaran que para esta investigación no se han realizado experimentos en seres humanos ni en animales.

Confidencialidad de los datos. Los autores declaran que han seguido los protocolos de su centro de trabajo sobre la publicación de datos de pacientes.

Derecho a la privacidad y consentimiento informado. Los autores han obtenido el consentimiento informado de los pacientes y/o sujetos referidos en el artículo. Este documento obra en poder del autor de correspondencia.

\section{Bibliografía}

1. Carol LA. Developmental dysplasia of the hip. En: Song KM, editor. Orthopaedic knowledge update: pediatrics 4. Rosemont, IL: AAOS; 2011. p. $159-67$.

2. Duncan ST, Bogunovic L, Baca G, Schoenecker PL, Clohisy JC. Are there sex-dependent differences in acetabular dysplasia characteristics? Clin Orthop Relat Res. 2015;473:1432-9.

3. Dobashi ET, Kiyohara RT, Matsuda MM, Milani C, Kuwajima SS, Ishida A. Inveterate dysplasic hip - a surgical treatment. Acta Ortop Bras. 2006;14:183-9.
4. Dega W. Schwierigkeiten in der chirurgischen reposition der veraltetenkongenitalen subluxation des hüftgelenkesbei Kindern. Beitr Orthop Traumatol. 1964;11:642-7.

5. Dega W. Selection of surgical methods in the treatment of congenital dislocation of the hip in children. Chir Narzadow Ruchu Ortop Pol. 1969;34:357-66.

6. Dega W. Transiliac osteotomy in the treatment of congenital hip dysplasia. Chir Narzadow Ruchu Ortop Pol. 1974;39:601-3.

7. Ozgur AF, Aksoy MC, Kandemir U, Karcaaltncaba M, Aydingoz U, Yazici $M$, et al. Does Dega osteotomy increase acetabular volume in developmental dysplasia of the hip? J Pediatr Orthop B. 2006;15:83-6.

8. Lepistö J, Armand M, Armiger RS. Periacetabular osteotomy in adult hip dysplasia-developing a computer aided real-time biomechanical guiding system (BGS). Suom Ortoped Traumatol. 2008;31:186-90.

9. Park MS, Chung CY, Lee SH, Cho TJ, Yoo WJ, Choi IH. Two-dimensional computed tomographic measurement of acetabulum reliability, validity, and limitation. J Pediatr Orthop. 2008;28:812-8.

10. Öhman C, Baleani M, Pani C, Taddei F, Alberghini M, Viceconti M, et al. Compressive behaviour of child and adult cortical bone. Bone. 2011; 49:769-76.

11. Smet MH, Marchal GJ, Baert AL, Van Hoe L, Van Cleynenbreugel J, Daniels $\mathrm{H}$, et al. Three-dimensional imaging of acetabular dysplasia: diagnostic value and impact on surgical type classification. Eur $\mathrm{J}$ Radiol. 2000;34:26-31.

12. Dandachli W, Islam SU, Liu M, Richards R, Hall-Craggs M, Witt J. Three-dimensional CT analysis to determine acetabular retroversion and the implications for the management of femoro-acetabular impingement. $J$ Bone Joint Surg (Br). 2009;91:1031-6.

13. Jia J, Li L, Zhang L, Zhao Q, Liu X. Three dimensional-CT evaluation of femoral neck anteversion, acetabular anteversion and combined anteversion in unilateral $\mathrm{DDH}$ in an early walking age group. Int Orthop. 2012;36:119-24.

14. Pinoit Y, May O, Girard J, Laffargue P, Ala Eddine T, Migaud H. Low accuracy of the anterior pelvic plane to guide the position of the cup with imageless computer assistance: variation of position in 106 patients. Rev Chir Orthop Reparatrice Appar Mot. 2007;93:455-60.

15. Humbert L, Carlioz H, Baudoin A, Skalli W, Mitton D. 3D Evaluation of the acetabular coverage assessed by biplanar X-rays or single anteroposterior X-ray compared with CT-scan. Comput Methods Biomech Biomed Engin. 2008;11:257-62.

16. Li L, Zhang L, Zhao Q, Wang E. Measurement of acetabular anteversion in developmental dysplasia of the hip in children by two- and three-dimensional computed tomography. J Int Med Res. 2009;37:567-75.

17. Jóźwiak M, Rychlik M, Musielak B, Chen BP, Idzio RM, Grzegorzewski A. An accurate method of radiological assessment of acetabular volume and orientation in computed tomography spatial reconstruction. BMC Musculoskelet Disord. 2015;16:42.

18. Larson CM, Moreau-Gaudry A, Kelly BT, Byrd JW, Tonetti J, Lavallee S, et al. Are normal hips being labeled as pathologic? A CT-based method for defining normal acetabular coverage. Clin Orthop Relat Res. 2015; 473:1247-54.

19. Zhao L, Zhao DY, Wang Z, Zhao L, Zhang JS, Huan Y, et al. 3D-CT for skeletal abnormality in developmental dislocation of the hip with a particular reference to surgical indications. World J Pediatr. 2006; 2:125-32.

20. Tannast M, Hanke M, Zheng G, Steppacher S, Siebenrock K. What are the radiographic reference values for acetabular under- and overcoverage? Clin Orthop Relat Res. 2015;473:1234-46.

21. Hansen BJ, Harris MD, Anderson LA, Peters CL, Weiss JA, Anderson AE. Correlation between radiographic measures of acetabular morphology with 3D femoral head coverage in patients with acetabular retroversion. Acta Orthop. 2012;83:233-9.

22. Akgül T, Göksan SB, Bilgili F, Valiyev N, Hürmeydan ÖM. Radiological results of modified Dega osteotomy in Tönnis grade 3 and 4 developmental dysplasia of the hip. J Pediatr Orthop B. 2014;23:333-8.

23. Rampal V, Klein C, Arellano E, Boubakeur Y, Seringe R, Glorion C, et al. Outcomes of modified Dega acetabuloplasty in acetabular dysplasia related to developmental dislocation of the hip. Orthop Traumatol Surg Res. 2014;100:203-7.

24. Chung CY, Park MS, Choi IH, Cho TJ, Yoo WJ, Lee KM. Morphometric analysis of acetabular dysplasia in cerebral palsy. J Bone Joint Surg (Br). 2006;88:243-7. 